\title{
Erkennen Sie eine HIV-Infektion?
}

\section{Alexandra Calmy, Jan Fehr, Pietro Vernazza, \\ Daniel Oertle}

Eidg. Kommission für sexuelle Gesundheit (EKSG)

Korrespondenz:

Dr. med. Daniel Oertle

Delegierter der FMH in der EKSG

Dennlerstrasse 25

CH-8047 Zürich
Wie die neueste Statistik des Bundesamtes für Gesundheit (BAG) zeigt, ist die Zahl der HIV-Neuinfektionen im letzten Jahr wieder deutlich angestiegen.

Umso wichtiger ist es, dass wir Ärztinnen und Ärzte ein Vorliegen einer möglichen sexuell übertragenen Krankheit frühzeitig erkennen und Patientinnen und Patienten einen HIV-Test anbieten.

Eine HIV-Infektion ist in den ersten drei Monaten nach erfolgter Infektion besonders leicht übertragbar. Viele Ansteckungen erfolgen in dieser Zeit der Primoinfektion, wenn die betroffene Person noch nicht diagnostiziert ist. Umso wichtiger ist es, eine Primoinfektion zu erkennen. Würden Sie eine HIV-Infektion erkennen? Eine Primoinfektion äussert sich wie ein grippaler Infekt. Deshalb ist bei Patienten mit einer fieberhaften Erkrankung, die an eine Mononukleose erinnert, immer an die Möglichkeit einer HIV-Primoinfektion zu denken. Atypische Symptome von Hauterkrankungen, neurologische Erscheinungen oder atypische Blutbefunde könnten an eine HIV-Infektion denken lassen: Immer dann ist ein HIV-Test anzubieten! Achtung: Unter Umständen reicht ein HIV-Suchtest (Antikörper/Antigen) nicht aus, und es kann nötig sein, eine HIV-PCR zu veranlassen, um eine Primoinfektion zu diagnostizieren. Wer in diesen Situationen - ähnlich wie bei Schwangeren oder Organspendern - vergisst, einen HIV-Test anzubieten, verletzt die ärztliche Sorgfaltspflicht.

Vor jedem HIV-Test ist das Einverständnis der Patientinnen und Patienten einzuholen. Eine entsprechende überarbeitete Richtlinie zu Provider Initiated Counselling and Testing (PICT) wird im Sommer 2013 vom BAG publiziert werden.

Wir Ärztinnen und Ärzte können durch richtiges Diagnostizieren weitere HIV-Infektionen verhindern helfen.

Darum: Nicht vergessen: HIV-Test!

\section{Reconnaîtriez-vous une infection au VIH?}

\section{Alexandra Calmy, Jan Fehr, Pietro Vernazza, \\ Daniel Oertle}

Commission fédérale pour la santé sexuelle (CFSS)
Correspondance: Dr Daniel Oertle Délégué de la FMH à la CFSS Dennlerstrasse 25 CH-8047 Zurich
Comme le montre la dernière statistique de l'Office fédéral de la santé publique (OFSP), le nombre de nouvelles infections au VIH a de nouveau fortement augmenté l'année dernière.

Il est donc d'autant plus important pour nous, médecins, de déceler suffisamment tôt l'existence d'une éventuelle maladie sexuellement transmissible et de proposer un test VIH à nos patientes et patients.

L'infection au VIH se transmet particulièrement facilement durant les trois premiers mois qui suivent la contamination. De nombreuses infections ont en effet lieu durant cette période dite de primo-infection, lorsque la personne concernée n'a pas encore été diagnostiquée. D'où l'importance de détecter à temps une primo-infection. Et vous, reconnaîtriezvous une infection au VIH? Les symptômes d'une primo-infection sont similaires à ceux d'une infection grippale. C'est pourquoi il faut toujours penser à une primo-infection au VIH en présence de patients souffrant d'une maladie accompagnée de fièvre et ressemblant à une mononucléose. Des symptômes atypiques comme des maladies cutanées, des troubles neurologiques ou des résultats sanguins atypiques pourraient faire penser à une infection au VIH: en présence de tels symptômes, il faut donc systématiquement proposer un test VIH. Attention: dans certains cas, un test de détection du VIH (anticorps/antigènes) ne suffit pas et il peut s'avérer nécessaire d'ordonner une PCR VIH pour diagnostiquer une primo-infection. Tout médecin qui oublie de proposer un test VIH dans ces situations (de même qu'en présence d'une femme enceinte ou d'un donneur d'organe) enfreint son devoir de diligence.

Avant chaque test VIH, il convient de solliciter l'accord de la patiente ou du patient. Des directives sur le «Provider Initiated Counselling and Testing (PICT)» seront publiées par l'OFSP en été 2013.

En posant le bon diagnostic, nous pouvons, nous médecins, contribuer à empêcher de nouvelles infections au VIH.

C'est pourquoi il ne faut jamais oublier le test VIH! 